\title{
Maximum Likelihood Estimation For SAR Interferometry
}

\author{
M. S. Seymour and I.G. Cumming \\ Dept. Of Electrical Engineering, U.B.C. \\ 2356 Main Mall, Vancouver, B.C., Canada, V6T 1Z4 \\ $\mathrm{T}: 604.822 .2872 \mathrm{~F}: 604.822 .5949$ \\ EMail: seymour@ee.ubc.ca, ianc@ee.ubc.ca
}

\begin{abstract}
Synthetic Aperture Radar (SAR) interferometry (InSAR) uses phase differences between overlapping SAR images to estimate terrain height and terrain height changes. In addition, the coherence magnitude between the images is often used as a measure of the quality of the data and the processing. By modeling the SAR image data as independent circular Gaussian random variates, we develop the maximum likelihood (ML) estimates for interferogram phase, coherence magnitude, and the variance of the underlying circular Gaussian distribution. We show that the ML estimate of interferogram phase is equivalent to the standard technique of computing the phase of averaged complex returns. The ML estimate of the coherence magnitude depends on the estimated interferogram phase. In comparison, the sample coherence magnitude estimate based on amplitudes alone is badly biased. We also derive the Cramer-Rao bound for each ML estimate. The ML estimate of interferogram phase is close to this bound for moderate to high coherence values. Similarly, the coherence magnitude is close to the bound for values of coherence greater than approximately $1 / 2$. For coherence magnitudes less than $1 / 2$, the ML estimate of coherence magnitude is biased for data samples sizes up to 16 samples.
\end{abstract}

\section{INTRODUCTION}

Interferograms are formed by multiplying a synthetic aperture radar (SAR) image by the complex conjugate of a second registered SAR image. When the images are correlated, the interferogram phase measures the difference in slant range distance phase (modulo $2 \pi$ ) to the common ground patch illuminated by both radar systems. By accounting for the missing $2 \pi$ quantities in the interferogram phase (phase unwrapping), accurate estimation of the slant range difference is possible. Through trigonometry, the estimated slant range difference accurately determines the location of pixel ground points (Graham, 1974). The coherence magnitude between the two images is a measure of the data quality and is a function the geometry of the interferometer formed by the two SAR systems, the terrain properties, and system parameters such as noise level (Zebker and Villasenor, 1992; Li and Goldstein, 1990; Bamler and Just, 1993).

We derive the ML estimate of interferogram phase, $\psi$; coherence magnitude, $\mu$; and variance of underlying images, $\sigma^{2}$ assuming a model of constant interferogram phase and independent pairs of data samples. We then compare the ML estimate

of coherence magnitude with a sample estimate derived from the definition of coherence magnitude (Prati and Rocca, 1992; Bamler and Just, 1993). The minimum error bound for unbiased estimates is given by the Cramer-Rao (CR) bound which we derive for each of these quantities. In addition, we compare the performance of the ML estimates with the CR bound. Finally, we summarize the results of our analysis.

0-7803-1497-2/94 \$4.00 ๔ 1994 IEEE

\section{MAXIMUM LIKELIHOOD ESTIMATION}

\section{Review of Maximum Likelihood Estimation}

Maximum likelihood estimation is a technique for estimating constant parameters associated with random observations or for estimating random parameters from random observations when the distribution of the parameters is unknown. The method picks the most likely set of parameters $(\theta)$ for a given set of observations $(z)$ by maximizing the probability that the observations came from the distribution defined by the parameters. To find the correct parameters, the likelihood function $f(z \mid \theta)$ (where $f(z \mid \theta)$ is the density function of the observations conditioned on the parameters) is maximized by solving

$$
\frac{\partial f(z \mid \theta)}{\partial \theta_{i}}=0 .
$$

For distributions which depend on exponential functions, it is computationally simpler to find the maximum of the log-likelihood function

$$
\frac{\partial \ln (f(z \mid \theta))}{\partial \theta_{i}}=0 .
$$

By solving for the extrema of the probability density function of the observations based on the parameters, the most likely set of parameters based on the observations is chosen.

\section{Estimates for SAR Interferometry}

Focused complex SAR images can be characterized as circular Gaussian random variables. For interferometry, two images of the same area are processed to extract the interferogram phase and coherence magnitude (optionally estimates of the data variance can also be made). The samples within each image are usually modeled as being uncorrelated since the bandwidth efficiency of SAR systems is usually quite high. However, individual returns in one image are correlated with the corresponding returns in the other image.

The probability density function (PDF) of the correlated returns can be modeled as the product of the PDF of individually correlated values between the two images

$$
\begin{gathered}
f\left(I_{1,1}, I_{2,1}, \theta_{1,1}, \theta_{2,1} \ldots, I_{1, N}, I_{2, N}, \theta_{1, N} \theta_{2, N} \mid \psi, \mu, \sigma^{2}\right) \\
=\prod_{k=1}^{N} f_{k}\left(I_{1, k}, \theta_{1, k}, I_{2, k}, \theta_{2, k} \mid \psi, \mu, \sigma^{2}\right),
\end{gathered}
$$

where (Goodman, 1984):

$$
\begin{gathered}
f_{k}\left(I_{1, k}, \theta_{1, k}, I_{2, k}, \theta_{2, k} \mid \psi, \mu, \sigma^{2}\right)= \\
\exp \left(-\left(I_{1, k}+I_{2, k}\right) /\left(2 \sigma^{2}\left(1-\mu^{2}\right)\right)\right) \\
\cdot \exp \left(2 \sqrt{I_{1, k} I_{2, k}} \mu \cos \left(\theta_{1, k}-\theta_{2, k}+\psi\right) /\left(2 \sigma^{2}\left(1-\mu^{2}\right)\right)\right) \\
\cdot 1 /\left(16 \pi^{2} \sigma^{4}\left(1-\mu^{2}\right)\right) .
\end{gathered}
$$

The definition of the log-likelihood functions follows easily as

$$
\begin{aligned}
\ln f(\cdot)= & -\sum_{k=1}^{N} \frac{I_{1, k}+I_{2, k}-2 \sqrt{I_{1, k} I_{2, k}} \mu \cos \left(\theta_{1, k}-\theta_{2, k}+\psi\right)}{2 \sigma^{2}\left(1-\mu^{2}\right)} \\
& -N \ln \left(16 \pi^{2} \sigma^{4}\left(1-\mu^{2}\right)\right) .
\end{aligned}
$$


ML estimates can be derived by setting the derivative of the likelihood or log-likelihood function with respect to the parameter of interest to zero and solving for the parameter. The maximum likelihood estimates (Seymour and Cumming, 1994) of interferogram phase, coherence magnitude, and variance are

$$
\begin{aligned}
\psi_{m i} & =-\arg \left(\sum_{k=1}^{N} x_{1, k} x_{2, k}^{*}\right), \\
\mu_{m i} & =\frac{2 b}{a} \\
\sigma_{m i}^{2} & =\frac{a-2 \mu b}{4 N\left(1-\mu^{2}\right)},
\end{aligned}
$$

where:

$$
\begin{aligned}
\arg (z) & =\text { returns the phase of the complex argument } z \\
x_{1, k} & =\text { complex magnitudes from the first image, } \\
x_{2, k} & =\text { complex magnitudes from the second image, } \\
N & =\text { the number of samples, } \\
z^{*} & =\text { complex conjugate of } z \\
a & =\sum_{k=1}^{N} I_{1, k}+I_{2, k}, \\
b & =\sum_{k=1}^{N} \sqrt{I_{1, k} I_{2, k}} \cos \left(\theta_{1, k}-\theta_{2, k}+\psi\right) .
\end{aligned}
$$

The ML estimate of $\psi$ is already well known (Rodriguez and Martin, 1992; Li and Goldstein, 1990). In general, $\psi$ will have to be estimated to calculate $\mu_{m l}$ and it appears that both $\mu$ and $\psi$ must be estimated to calculate $\sigma^{2}$. However, the sample estimate of $\sigma^{2}$ is equivalent to $\sigma_{m l}^{2}$ if $\mu_{m l}$ and $\psi_{m l}$ are used for the calculation (Seymour and Cumming, 1994). Simplifying the ML estimate of $\sigma^{2}$ after substituting the ML estimates of $\psi$ and $\mu$ into eqn. (8) yields the equivalent estimate

$$
\sigma_{m i}^{2}=\frac{\sum_{k=1}^{N} I_{1, k}+I_{2, k}}{4 N} .
$$

The sample estimate of $\mu$ may be defined as

$$
\mu_{s}=\frac{\left|\sum_{k=1}^{N} x_{1, k} x_{2, k}^{*} e^{j \Psi}\right|}{\sqrt{\left(\sum_{k=1}^{N} I_{1, k}\right)\left(\sum_{l=1}^{N} I_{2, l}\right)}}=\frac{|R+j I|}{\sqrt{C \cdot D}}
$$

while the ML estimate of $\mu$ is

$$
\mu_{m l}=\frac{\sum_{k=1}^{N} \sqrt{I_{1, k} I_{2, k}} \cos \left(\theta_{1, k}-\theta_{2, k}+\psi\right)}{\frac{1}{2} \sum_{k=1}^{N} I_{1, k}+I_{\ldots .}}=\frac{2 R}{C+D} .
$$

Although, it is not clear from these equations, the sample estimate is greater than or equal to the maximum likelihood estimate at all times.

Proof:

$$
\mu_{s} \stackrel{?}{\geq} \mu_{m i} \text {. }
$$

If one value is greater than or equal to the other, then surely

$$
\mu_{s}^{2} \stackrel{?}{\geq} \mu_{m l}^{2} .
$$

Substituting the definitions of the estimates in and expanding the squared terms:

$$
\frac{R^{2}+I^{2}}{C \cdot D} \stackrel{?}{\geq} \frac{4 R^{2}}{(C+D)^{2}} .
$$

Interchanging the denominators and simplifying gives

$$
\left(R^{2}+I^{2}\right)(C+D)^{2} \stackrel{?}{\geq} 4 R^{2} C D .
$$

Subtracting common terms and simplifying gives

$$
(C-D)^{2} R^{2}+(C+D)^{2} I^{2} \stackrel{?}{\geq} 0
$$

Since quadratic forms are always greater or equal to zero it must be true that

$$
\mu_{s} \geq \mu_{m l}
$$

Thus the sample estimate of $\mu$ is greater than or equal to the ML estimate. In the section on simulations we show that the sample estimate does not reach the ML estimate unless $\mu$ is close to one.

\section{CRAMER-RAO BOUND FOR InSAR}

\section{Review of Cramer Rao Bound}

The Cramer-Rao (CR) bound gives a lower bound on the variance of any unbiased estimate (Rice, 1988; Srinath and Rajasekaran, 1979). An estimate is said to be efficient if it achieves the bound. ML estimates achieve the bound asymptotically. We calculate the CR bound for each of the interferometric SAR parameters : $\psi, \mu$ and $\sigma^{2}$, and compare the performance of the estimates with the derived bounds.

The CR bounds are calculated from the inverse of the Fisher information matrix $\mathbf{J}$, with elements:

$$
J_{i, j}=-\mathrm{E}\left[\frac{\partial^{2} \ln f(z \mid \theta)}{\partial \theta_{i} \partial \theta_{j}}\right] .
$$

The minimum error variance or error covariance of the estimates is the corresponding element of the inverse of the Fisher information matrix:

$$
\mathrm{E}\left[\theta_{i} \theta_{j}\right] \geq \mathbf{\Psi}_{i, j}
$$

where

$$
\mathbf{\Psi}=\mathbf{J}^{-1} .
$$

A heuristic way of understanding the bound is to consider the distribution of the errors of ML estimates. Assuming the likelihood function is well behaved, it can be shown that the errors of ML estimates are distributed as approximately zero mean, normal random variables (Rice, 1988). The average curvature of a Gaussian distribution function is the inverse of the variance of the distribution. The CR bound identifies the average curvature of the distribution used to derive the ML estimate as the variance of the limiting normal distribution of the errors.

\section{Calculation of the CR Bounds for InSAR}

The Fisher Information matrix for InSAR is

$$
\mathbf{J}=-\mathbf{E}\left[\begin{array}{ccc}
\frac{\partial^{2} \ln f(\cdot)}{\partial \psi^{2}} & \frac{\partial^{2} \ln f(\cdot)}{\partial \psi \partial \mu} & \frac{\partial^{2} \ln f(\cdot)}{\partial \psi \partial \sigma^{2}} \\
\frac{\partial^{2} \ln f(\cdot)}{\partial \mu \partial \Psi} & \frac{\partial^{2} \ln f(\cdot)}{\partial \mu^{2}} & \frac{\partial^{2} \ln f(\cdot)}{\partial \mu \partial \sigma^{2}} \\
\frac{\partial^{2} \ln f(\cdot)}{\partial \partial \sigma^{2} \partial \psi} & \frac{\partial^{2} \ln f(\cdot)}{\partial \partial \sigma^{2} \partial \sigma^{2}} & \frac{\partial^{2} \ln f(\cdot)}{\partial \partial \sigma^{2} \partial \mu}
\end{array}\right] .
$$

Evaluating the expected values yields the following Fisher Information matrix for InSAR:

$$
\mathbf{J}=\left[\begin{array}{ccc}
\frac{2 N \mu^{2}}{1-\mu^{2}} & 0 & 0 \\
0 & \frac{2 N\left(1+\mu^{2}\right)}{\left(1-\mu^{2}\right)^{2}} & \frac{-2 N \mu}{\sigma^{2}\left(1-\mu^{2}\right)} \\
0 & \frac{-2 N \mu \mu}{\sigma^{2}\left(1-\mu^{2}\right)} & \frac{2 N}{\sigma^{4}}
\end{array}\right]
$$

The inverse of the Fisher information matrix is: 


$$
\Psi=\left[\begin{array}{ccc}
\frac{1-\mu^{2}}{2 N \mu^{2}} & 0 & 0 \\
0 & \Delta \frac{2 N}{\sigma^{4}} & \Delta \frac{2 N \mu}{\sigma^{2}\left(1-\mu^{2}\right)} \\
0 & \Delta \frac{2 N \mu}{\sigma^{2}\left(1-\mu^{2}\right)} & \Delta \frac{2 N\left(1+\mu^{2}\right)}{\left(1-\mu^{2}\right)^{2}}
\end{array}\right]
$$

where:

$$
\Delta=\frac{\sigma^{4}\left(1-\mu^{2}\right)^{2}}{4 N^{2}} \text {. }
$$

Simplifying the expression for the inverse matrix yields the CR lower bound for the measured quantities:

$$
\begin{aligned}
\mathrm{E}\left[\left(\psi-\psi_{o}\right)^{2}\right] & >\frac{1-\mu^{2}}{2 N \mu^{2}}, \\
\mathrm{E}\left[\left(\mu-\mu_{o}\right)^{2}\right] & >\frac{\left(1-\mu^{2}\right)^{2}}{2 N}, \\
\mathrm{E}\left[\left(\sigma^{2}-\sigma_{o}^{2}\right)^{2}\right] & >\frac{\sigma^{4}\left(1+\mu^{2}\right)}{2 N} .
\end{aligned}
$$

Note that the error covariance between $\psi$ and both $\mu$ and $\sigma^{2}$ is 0 .

\section{SIMULATIONS}

Although the CR bound is reached asymptotically by ML estimates, when a small number of samples are used or the signalto-noise ratio is low, the CR bound may not be attained. To verify the performance of the ML estimates simulations were performed. 10,000 realizations of $\mathrm{N}=8$ and $\mathrm{N}=16$ interferomet ric complex circular Gaussian data pairs were generated for values of $\mu$ between 0.05 and 0.95 . Each data pair was generated according to

$$
y_{1}=x+n 1 \quad y_{2}=x \cdot e^{j \phi}+n 2,
$$

where:

$$
\begin{aligned}
y_{i} & =\text { unit variance circular Gaussian variables } \\
n_{i} & =\text { additive circular Gaussian white noise } \\
x & =\text { circular Gaussian random variable } \\
\phi & =\text { simulated interferometric phase. }
\end{aligned}
$$

The relative variance of $x$ and $n_{i}$ was varied to achieve the desired value of $\mu$ for the simulation. The angle variable $\phi$ was generated using a uniform distribution. The angle was common to each $N$ sample realization but varied between realizations for a specific value of $\mu$. For each realization, the ML estimate of $\psi$, $\mu$, and $\sigma^{2}$, as well as the sample $\mu$ estimate was calculated. The performance of the ML estimate as a function of $\mu$ is shown in Figure (1) through Figure (8). Each plot shows the variance, the $\mathrm{CR}$ bound, and the mean error of the estimates. The variance and CR bounds are defined by the axis on the left side of each plot while the mean error is defined by the right axis of each plot.

For the estimated interferometric angle, the variance nearly reaches the $\mathrm{CR}$ bound at high values of $\mu$ and approaches the bound more closely as the number of samples increases. As $\mu$ decreases the variance of the estimates increases and then decreases until reaching the bound when $\mu \approx 0.35$. Normally, unbiased estimates can never have less variance than the $\mathrm{CR}$ bound. However the variance of the ML estimate does become less than the bound for $\mu<0.35$ because we calculate the plase error in the sense of phase wrapping. That is, the phase errors are always taken into the principal phase domain from $-\pi$ to $\pi$. For example, an estimated phase of $-9 \pi / 10$ is only in error by $3 \pi / 10$ from an actual phase value of $8 \pi / 10$. The CR bound uses the local curvature of the distribution function to calculate the minimum variance of the estimate and therefore does not take into account the wrapping of phase estimates into the principal phase domain of $\pi$ to $-\pi$.

The ML estimates of $\mu$ are fairly close to the CR bound for $\mu>0.5$. For $\mu<0.5$, the ML estimate of $\mu$ is biased and therefore, the CR bound is not valid. The sample estimate of $\mu$ is biased unless the value of $\mu$ is close to one.

The ML estimate of $\sigma^{2}$ is above the bound for most of the values of $\mu$ except when $\mu$ is close to zero or one.

\section{CONCLUSION}

The maximum likelihood estimates for the interferometric SAR parameters of interferogram phase, $\psi$; coherence magnitude, $\mu$; and image sample variance, $\sigma^{2}$ were derived under the assumptions of independent observations and constant interferogram phase. In addition, the Cramer-Rao bound which defines the minimum variance of any unbiased estimate was also calculated for each parameter. The performance of InSAR systems using pairwise correlated image samples will be well modeled by the results of this paper.

\section{REFERENCES}

Bamler, R. and Just, D. (1993). Phase statistics and decorrelation in SAR interferograms. In IGARSS'93, pages 980-984.

Goodman, J. (1984). Statistical properties of laser speckle patterns. In Dainty, J., editor, Laser Speckle and Related Phe nomena, pages 9-75. Springer Verlag.

Graham, L. C. (1974). Satellite interferometer radar for topographic mapping. Proceedings of the IEEE, 62(6):763-768.

Li, F. K. and Goldstein, R. (1990). Studies of multibaseline spaceborne interferometric synthetic aperture radars. IEEE Transactions on Geoscience and Remote Sensing, 28(1):8897.

Prati, C. and Rocca, F. (1992). Range resolution enhancement with multiple SAR surveys combination. In IGARSS'92, pages 15761578 , Houston, Texas.

Rice, J. A. (1988). Mathematical Statistics and Data Analysis. Wadsworth and Brooks/Cole Advanced Books and Software, Pacific Grove California.

Rodriguez, E. and Martin, J. M. (1992). Theory and design of interferometric synthetic aperture radars. IEE ProceedingsF, 139(2):147-159.

Seymour, M. and Cumming, I. (1994). Maximum Likelihood Estimation and Cramer-Rao Bounds for SAR Interferometry. Technical report, University of British Columbia.

Srinath, M. and Rajasekaran, P. (1979). An Introduction to Statistical Signal Processing With Applications. John Wiley and Sons. Inc.

Zebker, H. and Villasenor, J. (1992). Decorrelation in interferometric radar echoes. IEEE Transactions Geoscience and Remote Sensing, Vol 30(5):950-959.

The authors gratefully acknowledge the financial support of the Natural Sciences and Research Council (NSERC), MacDonald Dettwiler and Associates, the British Columbia Science Council, and the British Columbia Advanced Systems Institute. 


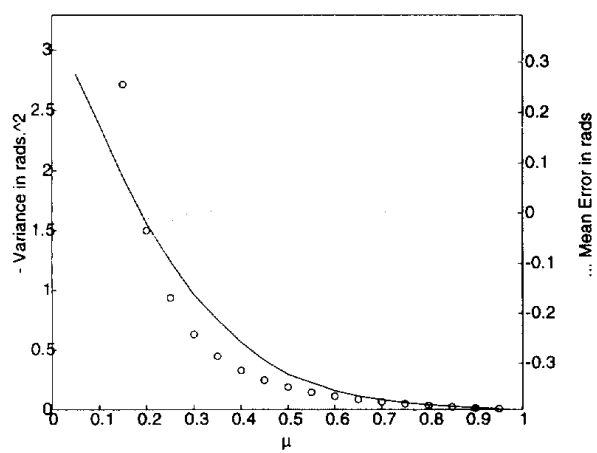

Figure 1: ML estimate of $\psi$ : plot of variance, mean error and the CR bound for $\mathrm{N}=8$ ( 0 is $\mathrm{CR}$ Bound).

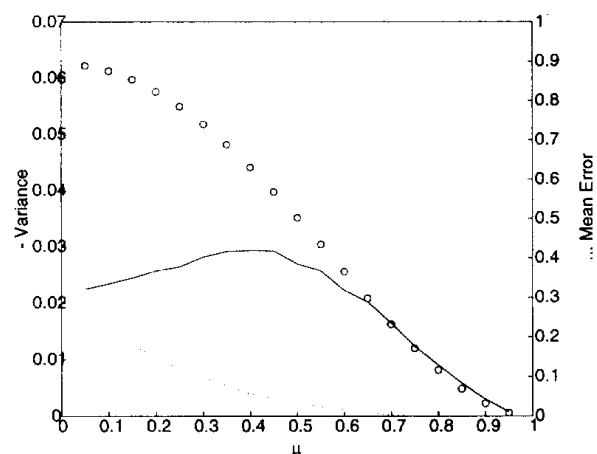

Figure 3: ML estimate of $\mu$ : plot of variance, mean error and the $\mathrm{CR}$ bound for $\mathrm{N}=8$ ( $\mathrm{o}$ is $\mathrm{CR}$ Bound)

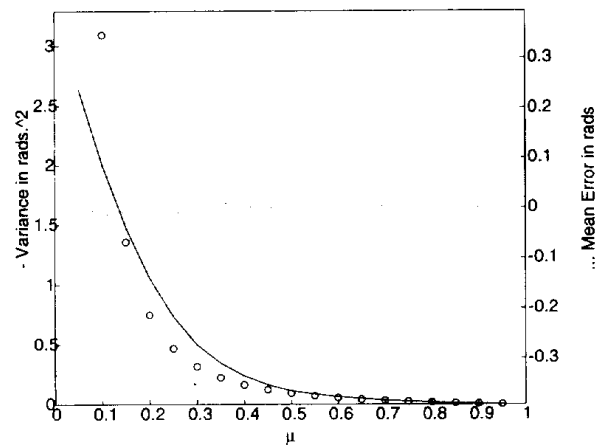

Figure 5: ML estimate of $\psi$ : plot of variance, mean error and the CR bound for $\mathrm{N}=16$ ( $\mathrm{o}$ is $\mathrm{CR}$ Bound).

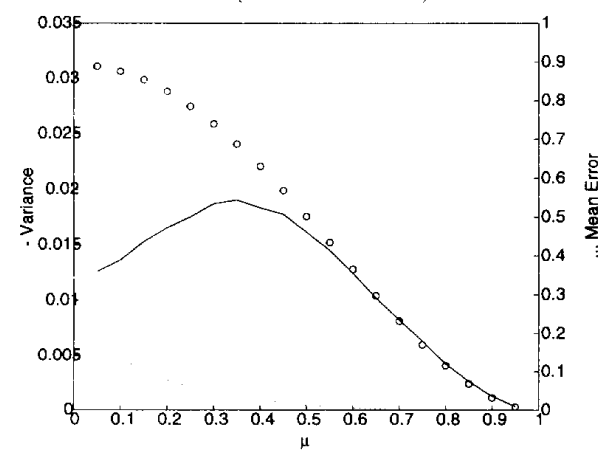

Figure 7 : ML estimate of $\mu$ : plot of variance, mean error and the CR bound for $\mathrm{N}=16$ ( $\mathrm{o}$ is $\mathrm{CR}$ Bound).

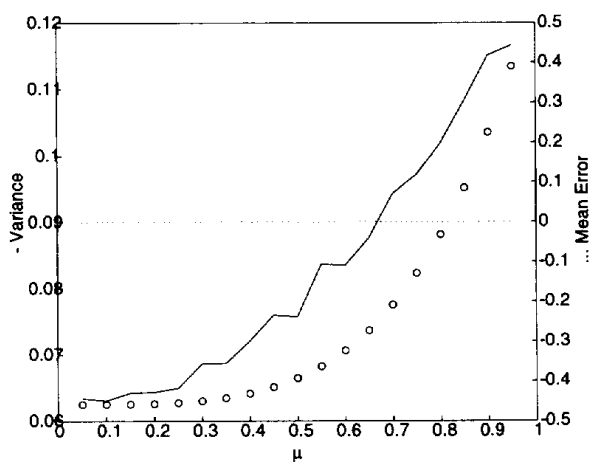

Figure 2: ML estimate of $\sigma^{2}$ : plot of variance, mean error and the CR bound for $\mathrm{N}=8$ ( $\mathrm{o}$ is CR Bound).

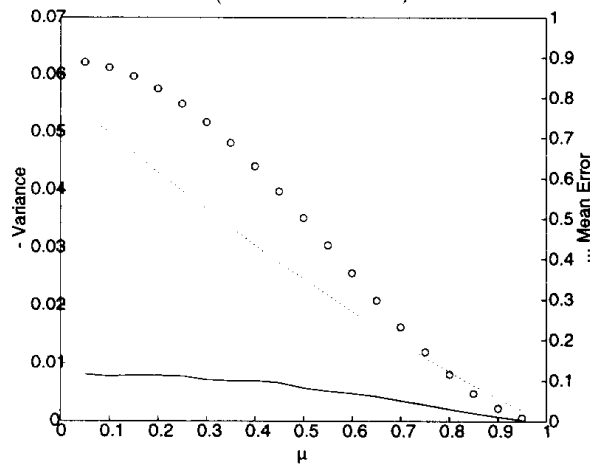

Figure 4: Sample $\mu$ : plot of variance, mean error and the $\mathrm{CR}$ bound for $\mathrm{N}=8$ ( $\mathrm{o}$ is $\mathrm{CR}$ Bound).

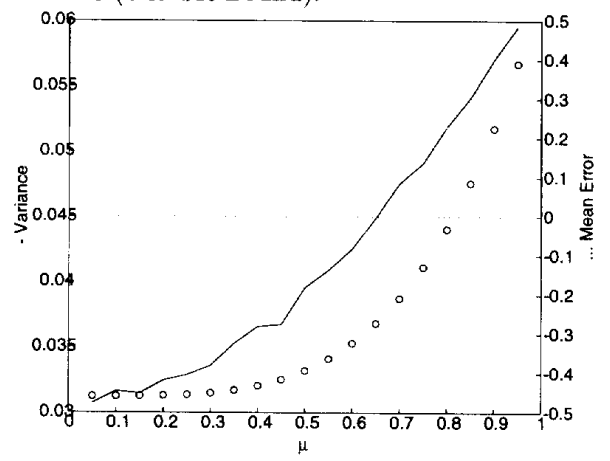

Figure 6: ML estimate of $\sigma^{2}$ : plot of variance, mean error and the CR bound for $\mathrm{N}=16$ ( $\mathrm{o}$ is CR Bound).

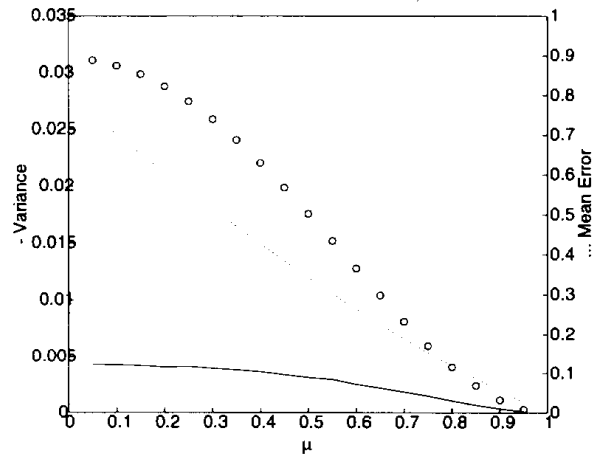

Figure 8: Sample $\mu$ : plot of variance, mean error and the CR bound for $\mathrm{N}=16$ ( $\mathrm{o}$ is CR Bound). 